\title{
Synchronization of Lu Chaotic System by Using Linear Feedback Control and Adaptive Control Methods
}

\author{
Grienggrai Rajchakit
}

\begin{abstract}
This paper treats the chaos synchronization problem of chaotic Lu system via single variable information of the master system. By stability theory of cascade-connection system, the derived controller is featured as follows: (a) only single variable information of the master system is needed to transmit to slave system for synchronization; (b) the pre-knowledge of upper bound of the trajectory of the master system is eliminated; (c) the controller is linear feedback. Finally, the effectiveness of the proposed control law is also illustrated by the numerical simulation on Matlab environment as well as rigorous mathematical proof.
\end{abstract}

Keywords - Lu Chaotic System, Single Variable, Chaos Synchronization, Nonlinear Feedback.

\section{INTRODUCTION}

In various application fields such as engineering, physics, chemistry, biology, and economy, we encounter phenomena that undergo spatial and temporal evolution. An important tool in modeling and analyzing these phenomena is the study of dynamical systems and chaos. The applications of dynamical systems and chaos include chaos control and synchronization, electronic circuits, secure communications, image encryption, cryptography, and neuroscience research. In the mid-1960s, Chua proposed a two-terminal resistor with a piecewisecontinuous voltage-current characteristic called Chua's diode and the resulting circuit is the well-known Chua's circuit. This motivated many authors to develop different nonlinear circuits and utilize these circuits in studying nonlinear phenomena and chaos. A modification to the ADVP circuit by adding a resistor in parallel with the inductor in the ADVP circuit is proposed in with the advantage that all the dynamics of the ADVP oscillator are displayed in a small range when varying the new system's parameter. The fourth element in electronic circuits, called the memristor, was postulated by Chua in 1971. It is a two terminal element, in which the magnetic flux $\phi$ between the terminals is a function of the electric charge $q$ that passes through the device. The memristor took its place alongside the rest of the more familiar circuit elements the resistor, the capacitor, and the inductor, when Williams and coworkers fabricated a solid state implementation of this proposed element. Several important applications of memristors have Science, Maejo University, Chiang Mai 50290, Thailand been reported which include ultra dense semi-non-volatile memories and learning networks that require a synapse-like function. Also, one can obtain high frequency chaotic oscillators, which have great potential for applications in secure communication by using nano-scale devices like the memristor.

Chaos control and synchronization have been intensively investigated during last decade [1-3] and still have attracted increasing attention in recent years. Chaos synchronization has many potential applications in secure communication, laser physics, chemical reactor, biomedical and so on. Up to now, numerous methods has been proposed to cope with the chaos synchronization, such as back stepping design method [3], adaptive design method [4], impulsive control method [5], sliding mode control method [6,7], and other control methods [8-14]. The objectives of this paper are as follows. Firstly, to give sufficient conditions of parameters that make equilibrium points of the Lu's system to be asymptotically stable by using linear feedback control and adaptive control methods. Finally, we investigate adaptive synchronization for the Lu's system using active control.

Consider two nonlinear systems:

$\dot{x}=f(t, x)$

$\dot{y}=g(t, y)+u(t, x, y)$

Where $x, y \in \mathfrak{R}^{n}, f, g \in C^{r}\left[\mathfrak{R}^{+} \times \mathfrak{R}^{n}, \mathfrak{R}^{n}\right]$,

$u \in C^{r}\left[\mathfrak{R}^{+} \times \mathfrak{R}^{n} \times \mathfrak{R}^{n}, \mathfrak{R}^{n}\right], r \geq 1, \mathfrak{R}^{+}$is the set of nonnegative real numbers. Assume that (1) is the drive system, (2) is the response system, and $u(t, x, y)$ is the control vector.

\section{CHAOS SYNCHRONIZATION}

Definition 1. Response system and drive system are said to be synchronic if for any initial conditions $x\left(t_{0}\right), y\left(t_{0}\right) \in \mathfrak{R}^{n}$,

$$
\lim _{t \rightarrow+\infty}\|x(t)-y(t)\|=0
$$

In this section, we assume that there are two Lu's systems such that the drive system (with the subscript 1) drives the response 
system (with the subscript 2). The drive and response systems are given, respectively, by

$$
\begin{aligned}
& \dot{x}_{1}=a\left(y_{1}-x_{1}\right) \\
& \dot{y}_{1}=-x_{1} z_{1}+c y_{1} \\
& z_{1}=x_{1} y_{1}-b z_{1}
\end{aligned}
$$

and

$$
\begin{aligned}
& \dot{x}_{2}=a\left(y_{2}-x_{2}\right)+u_{1} \\
& \dot{y}_{2}=-x_{2} z_{2}+c y_{2}+u_{2} \\
& \dot{z}_{2}=x_{2} y_{2}-b z_{2}+u_{3}
\end{aligned}
$$

We have introduced three control functions

$u_{1}(t), u_{2}(t), u_{3}(t)$ in (4). Our goal is to determine the control functions $u_{1}(t), u_{2}(t), u_{3}(t)$. We define the errors system as the difference between system (3) and the controlled system (4). Let us define the state errors between the response system (4) and the drive system (3) as

$$
\begin{aligned}
& e_{x}=x_{2}-x_{1} \\
& e_{y}=y_{2}-y_{1} \\
& e_{z}=z_{2}-z_{1}
\end{aligned}
$$

By subtracting (3) from (4) and by using the notation in (5), we have

$$
\begin{aligned}
& \dot{e}_{x}=a\left(e_{y}-e_{x}\right)-2 a^{2} e_{x}+u_{1}(t) \\
& e_{y}=x_{1} z_{1}-x_{2} z_{2}-4 c^{4} e_{y}+u_{2}(t) \\
& e_{z}=x_{2} y_{2}-x_{1} y_{1}-9 b^{2} e_{z}+u_{3}(t)
\end{aligned}
$$

We define active control functions $u_{1}(t), u_{2}(t), u_{3}(t)$ as follows

$$
\begin{aligned}
& u_{1}(t)=V_{1}(t)-2 a^{2} e_{x} \\
& u_{2}(t)=V_{2}(t)-4 c^{4} e_{y} \\
& u_{3}(t)=V_{3}(t)-9 b^{2} e_{y} .
\end{aligned}
$$

Hence, the error system (6) becomes

$$
\begin{aligned}
& \dot{e}_{x}=V_{1}(t)-2 a^{2} e_{x} \\
& \dot{e}_{y}=V_{2}(t)-4 c^{4} e_{y} \\
& \dot{e}_{z}=V_{3}(t)-9 b^{2} e_{z}
\end{aligned}
$$

The error system (7) is a linear system with control input $V_{1}(t), V_{2}(t), V_{3}(t)$ as functions of the error $e_{x}, e_{y}, e_{z}$. There are a number of possible choices for the controls $V_{1}(t), V_{2}(t), V_{3}(t)$. We choose

$$
\left[\begin{array}{l}
V_{1}(t) \\
V_{2}(t) \\
V_{3}(t)
\end{array}\right]=A\left[\begin{array}{l}
e_{x} \\
e_{y} \\
e_{z}
\end{array}\right],
$$

Where $A$ is a $3 \times 3$ constant matrix. Let the matrix $A$ is chosen in the following form

$$
A=\left(\begin{array}{ccc}
-2 a^{2} & 0 & 0 \\
0 & -4 c^{4} & 0 \\
0 & 0 & -9 b^{2}
\end{array}\right)
$$

Since eigenvalues of $A$ are $-2 a^{2},-4 b^{4},-9 c^{2}$, where $a, b, c$ are positive constants. It follows that $e_{x}, e_{y}, e_{z}$ converge to zero as $t$ tends to $+\infty$ and hence the Lu's systems (3) from (4) are synchronic.

\section{NUMERICAL SIMULATIONS}

Fourth-order Runge-Kutta integration method is used to solve two systems of differential Equations. (3) and (4) with time step size 0.1 . The values of parameters in (3) are chosen as $a=1, b=1, c=1$ to ensure the chaotic behavior of Lu's systems. The initial conditions of the drive system are $x_{1}(0)=0.5, y_{1}(0)=0.1, z_{1}(0)=0.2$ and the initial conditions of the response system are $x_{2}(0)=0.5, y_{2}(0)=0.9, z_{2}(0)=0.4$. Thus, the initial values of the error system are $e_{x}(0)=-0.23, e_{y}=0.96, e_{z}=0.69$. Fig. $1-3$ show that the synchronization is occurred after applying active control at Fig. 4 show that the state errors $\left(e_{x}, e_{y}, e_{z}\right)$ of Lu's systems of equations with the active control activated. 


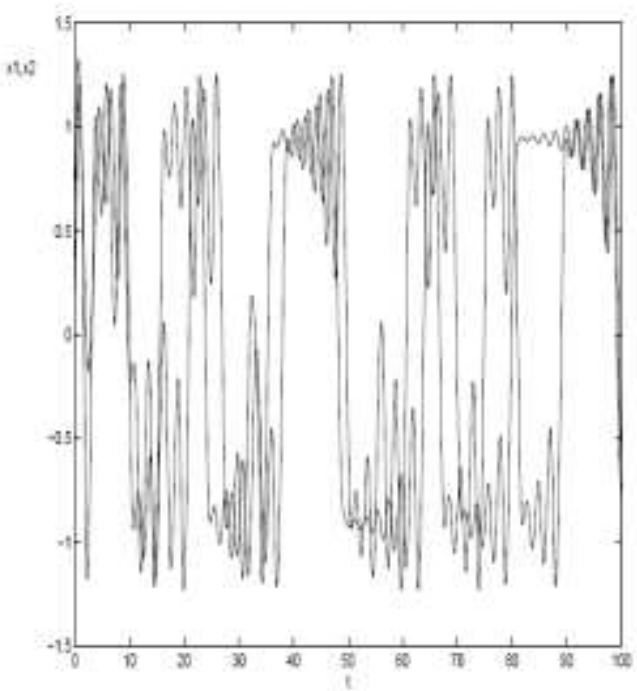

Figure 1: The state $x_{1}, x_{2}$ of the coupled Lu's systems of equations with the active control activated.

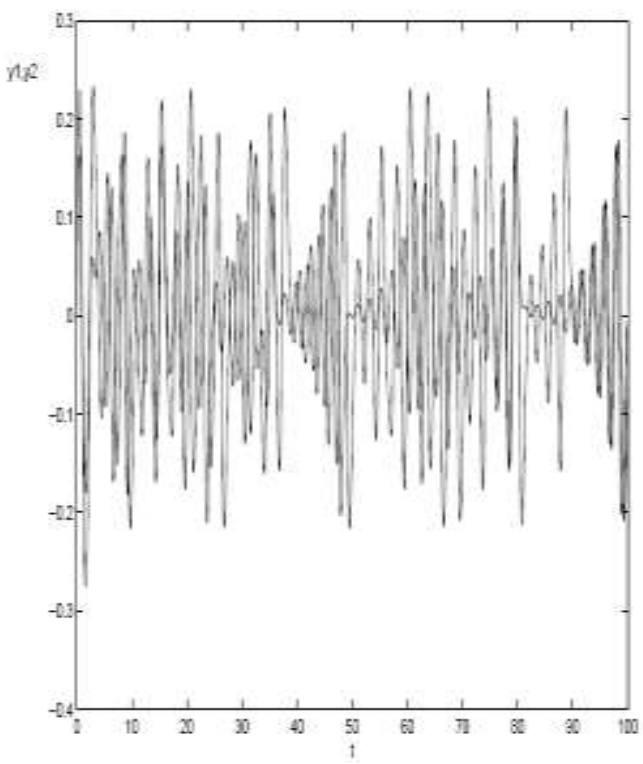

Figure 2: The state $y_{1}, y_{2}$ of the coupled Lu's systems of equations with the active control activated.

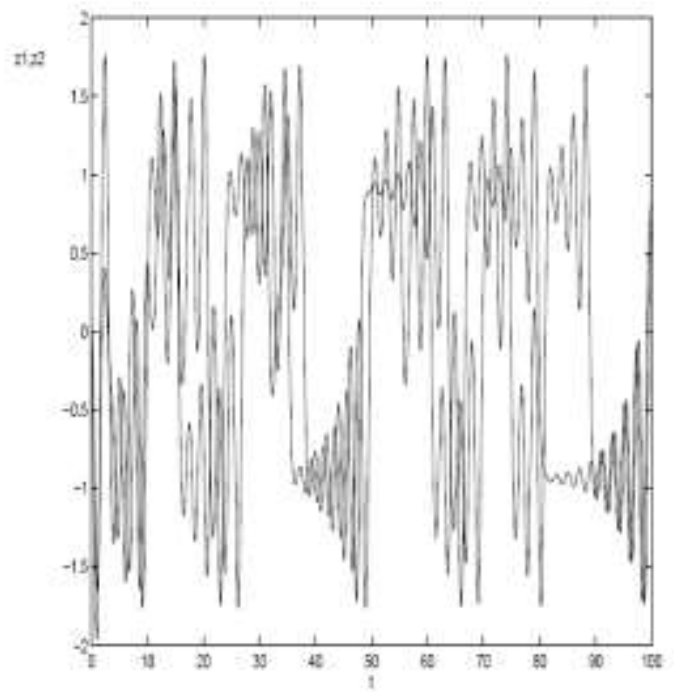

Figure 3: The state $z_{1}, z_{2}$ of the coupled Lu's systems of equations with the active control activated.

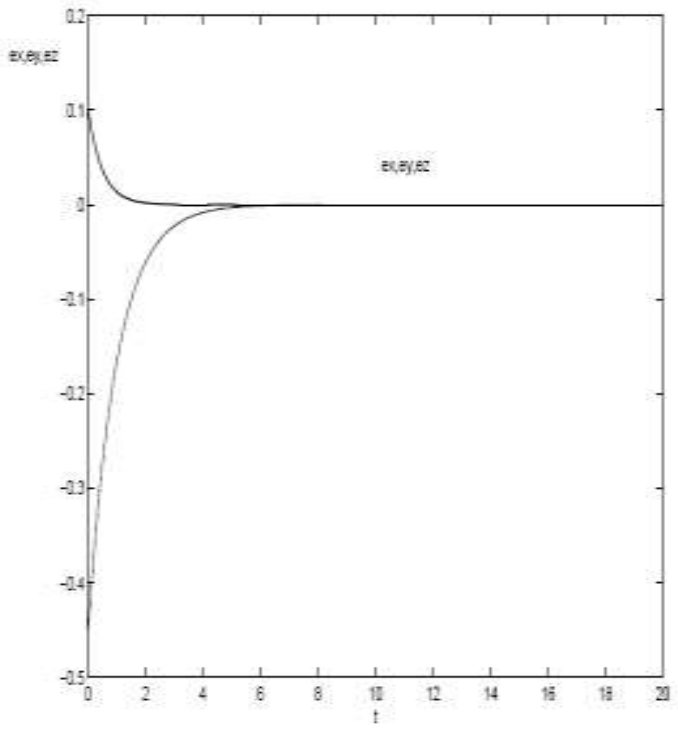

Figure 4: The state errors $\left(e_{x}, e_{y}, e_{z}\right)$ of the coupled Lu's systems of equations with the active control activated.

\section{CONCLUSION}

In this paper, we first give sufficient conditions for stability of equilibrium points of linear feedback controls which control the chaotic behavior of Lu's system to its equilibrium points. Finally, we give active controls which synchronize Lu's system. Numerical Simulations are also given to verify results we obtained. 


\section{ACKNOWLEDGMENT}

This work was supported by the Office of Agricultural Research and Extension Maejo University Chiangmai Thailand and the Higher Education Commission and Faculty of Science, Maejo University, Thailand.

\section{REFERENCES}

[1] K. Abdelwahab and R.B. Guenther, An introduction to numerical methods a MATLAB approach, Chapman and Hall/CRC, New York, 2002.

[2] S. Barnett and R.G. Cameron, Introduction to Mathematical Control Theory, Oxford, Clarendon Press, 1985.

[3] J. Lu, G. Chen, A new chaotic attractor coined, Int. J. Bifurc. Chaos 12 (2002) 659-661.

[4] V.N. Phat, Constrained Control Problems of Discrete Processes, World Scientific Publisher,Singapore-NewJersey-London,1996.

[5] V.N. Phat, Introduction to Mathematical Control Theory, Hanoi National University Publisher,Hanoi, 2001.

[6] K. Ratchagit, "Asymptotic Stability of Delay Difference System of the Hopfield Neural Networks via Matrix Inequalities and Application", International Journal of Neural Systems, Vol. 17, No. 5, pp. 425-430 (2007).

[7] K. Ratchagit, "Asymptotic Stability of Delay-Difference Control System of Hopfield Neural Networks via Matrix Inequalities", International Mathematical Forum, Vol. 4, No. 28, pp. 1405-1411 (2009).

[8] K. Ratchagit, "Asymptotic Stability of Time Varying Delay-Difference System of Cellular Neural Networks with Time-Varying Delay via Matrix Inequalities and Applications", Int. J. of Appl. Math and Mech, Vol. 5, No. 3, pp. 11-21 (2009).

[9] K. Ratchagit, "Asymptotic Stability of Nonlinear Delay-Difference Control System with Time-Varying Delay via Matrix Inequalities", Applied Mathematical Sciences, Vol. 3, No. 30, pp. 1473-1478 (2009).

[10] K. Ratchagit, M. Ratchagit, in: International Conference on Circuits, System and Simulation, IPCSIT, vol. 7, 2011, pp. 188-192.

[11] F. Zhang, C. Mu, X. Li, International Journal of Bifurcations and Chaos 22 (1) (2012), http://dx.doi.org/10.1142/S0218127412500150.

[12] I. Li, G. Chen, International Journal of Bifurcation and Chaos 16 (10) (2006) 3035.

[13] V.A. Yakubovich, G.A. Leonov, A.Kh. Gelig, Stability of Stationary Sets in Control Systems with Discontinuous Nonlinearities, World-Scientific, 2004, 334 pp.

[14] G.A. Leonov, Journal of Applied Mathematics and Mechanics 74 (2) (2010) 135.

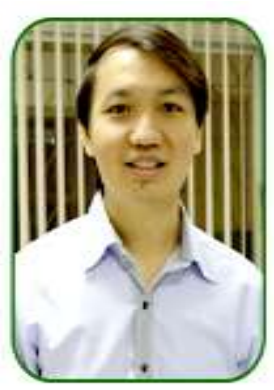

Grienggrai Rajchakit received his MSc degrees in applied mathematics at the Chiang Mai University, Thailand in 2001 and PhD degrees in applied mathematics at the King Mongkut's University, Thailand in 2007, respectively. He is the author/co-author of 89 refereed journal papers. In 2007 he joined the Department of Mathematics, Maejo University Thailand as a Lecturer, where he became an Assistant Professor in 2013. His research areas include mathematical analysis, stability analysis, difference equations, neural networks. 